\title{
A Guideline-Based Decision Tree Achieves Better Glucose Control with Less Hypoglycemia at 3 Months in Chinese Diabetic Patients
}

\author{
Yingying Luo $\cdot$ Hong $\mathrm{Wu} \cdot$ Xiyang Liao · Tingting Zhao • \\ Nan Cui · Aihua Li · Xingzhi Sun · Puhong Zhang · Yahua Huang • \\ Xia Zhang · Huiqiu Yin · Linong Ji
}

Received: January 13, 2021 / Accepted: May 7, 2021 / Published online: May 29, 2021

(C) The Author(s) 2021

\section{ABSTRACT}

Introduction: China has the world's largest diabetes epidemic and has been facing a serious shortage of primary care providers for chronic diseases including diabetes. To help primary care physicians follow guidelines and mitigate the workload in primary care communities in China, we developed a guideline-based decision tree. This study aimed to validate it at 3 months with real-world data.

Methods: The decision tree was developed based on the 2017 Chinese Type 2 Diabetes (T2DM) guideline and 2018 guideline for primary care. It was validated with the data from

Supplementary Information The online version contains supplementary material available at https:// doi.org/10.1007/s13300-021-01075-1.

Y. Luo · L. Ji $(\bowtie)$

Department of Endocrinology and Metabolism, Peking University People's Hospital, Beijing, China e-mail: jiln@bjmu.edu.cn

Y. Luo $\cdot$ L. Ji

Peking University Diabetes Center, Beijing, China

H. Wu · N. Cui · A. Li · Y. Huang · X. Zhang · H. Yin Sanofi (China) Investment, Shanghai, China

X. Liao $\cdot$ T. Zhao $\cdot$ X. Sun

Ping An Healthcare Technology, Beijing, China

P. Zhang

The George Institute for Global Health at Peking University Health Science Center, Beijing, China two registry studies: the NEW2D and ORBIT studies. Patients' data were divided into two groups: the compliance and non-compliance group, depending on whether the physician's prescription was consistent with the decision tree or not. The primary outcome was the difference of change in HbA1c from baseline to 3 months between the two groups. The secondary outcomes included the difference in the proportion of patients achieving HbA1c $<7 \%$ at 3 months between the two groups, the incidence of self-reported hypoglycemia at 3 months, and the proportion of patients (baseline $\mathrm{HbA1c} \geq 7 \%$ ) with a HbA1c reduction $\geq 0.3 \%$. The statistical analysis was performed using linear or logistic regression with inverse probability of treatment weighting with adjustments of confounding factors.

Results: There was a $0.9 \%$ reduction of $\mathrm{HbA1c}$ in the compliance group and a $0.8 \%$ reduction in the non-compliance group $(P<0.001)$; $61.1 \%$ of the participants in the compliance group and $44.3 \%$ of the participants in the noncompliance group achieved a HbA1c level $<7 \%$ at 3 months $(P<0.001)$. The hypoglycemic events occurred in $7.1 \%$ of patients in the compliance group vs. $9.4 \%$ in the non-compliance group $(P<0.001)$.

Conclusion: The decision tree can help physicians to treat their patients so that they achieve their glycemic targets with fewer hypoglycemic risks. (http://www.clinicaltrials.gov NCT01525 693 \& NCT01859598). 
Keywords: Decision tree; Guideline; Diabetes; Glucose control; Hypoglycemia

\section{Key Summary Points}

\section{Why Carry Out This Study?}

China has the world's largest diabetes epidemic and has been facing a serious shortage of primary care providers for chronic diseases including diabetes. To help primary care physicians follow guidelines and mitigate the workload in primary care communities in China, we developed a guideline-based decision tree. We hypothesized that this decision tree could aid physicians in following the guidelines and helping more patients achieve their HbA1c target with fewer hypoglycemic events. This study aimed to validate the decision tree at 3 months with real-world data

\section{What Was Learned from the Study?}

Patients following the decision tree have a greater $\mathrm{HbA1c}$ reduction at 3 months from baseline and are more likely to achieve HbA1c $<7 \%$, more likely to have HbA1c reduction $\geq 0.3 \%$ from baseline, and less likely to experience hypoglycemia at 3 months. The decision tree can help physicians, especially primary care physicians, to treat their patients and reach the glycemic target with fewer hypoglycemic risks

\section{DIGITAL FEATURES}

This article is published with digital features, including a summary slide, to facilitate understanding of the article. To view digital features for this article go to https://doi.org/10.6084/ m9.figshare.14519025.

\section{INTRODUCTION}

Type 2 diabetes mellitus (T2DM) is a progressive metabolic disease that causes a huge disease burden with high incidence and prevalence [1-3]. China has the largest number of diabetes patients, and the prevalence of T2DM has been estimated to be $12.8 \%$ in Chinese adults, with $43.3 \%$ of these being aware of diabetes, $49 \%$ receiving treatment, and only $49.4 \%$ of those treated achieving the HbA1c target $<7 \%$ [4]. The situations are even worse in rural areas. The prevalence of total diabetes is higher in men than in women. The total number of patients with diabetes in mainland China is estimated to be 129.8 million (70.4 million men and 59.4 million women) [4]. The Chinese Center for Disease Control and Prevention (China CDC) [5] also reported that, from 2000 to 2016, the number of diabetes cases in China increased by $62.7 \%$. As we look to the future, the burden of diabetes will steadily increase and China will face increasing health challenges due to more sedentary lifestyle changes and the aging population.

WHO [6] recognizes that Primary Health Service is the most efficient and cost-effective way to meet peoples' health needs. There is strong international evidence that a health system centered around community health services staffed by well-trained General Practitioners (GPs), close to where people live, is the most effective and efficient way to provide good quality health care to the whole population.

The Chinese government has put health at the center of the country's entire policy-making machinery [6]. Further development of Primary Health Care in China can play a valuable role in the prevention and management of chronic diseases. On September 8, 2015, the Chinese General Office of the State Council issued a document called "Guiding Opinions on Promoting the Construction of a Graded Diagnosis and Treatment System" [7]. According to this guidance, the graded diagnosis and treatment system will be actively promoted at the whole country level. Strengthening the primary care system, optimizing the graded diagnosis and treatment system, building up the formal 
referral medical system, and pushing chronic disease care from the specialists to general physicians are inevitable trends in China.

However, China has been facing a serious shortage of primary care providers for chronic disease management in the primary care setting. For primary care physicians to make the right decision efficiently is becoming a key factor for the successful achievement of T2DM management $[8,9]$. To help primary care physicians follow clinical guidelines to manage type 2 diabetes (T2DM) patients and mitigate the workload, we developed a guideline-based decision tree, which is a digital application to assist with guideline-based treatment decision making. We hypothesized that this decision tree could help physicians treat more patients and achieve their HbA1c targets with fewer hypoglycemic events by the following the guidelines. This study aimed to validate the clinical value of this decision tree at 3 months with data from real-world practice.

\section{METHODS}

\section{Rule of the Clinical Decision Tree}

The decision tree was designed by simulating clinical practice. It was built with the following patient information: diagnosis, medication, adverse events, and laboratory data combined with the rules of drug contraindication, medication selection, and request for referral. Starting at the tree root, patients were split into two processes based on whether they had been treated or not. Then, the patients were further separated into different branches by using HbA $1 \mathrm{c}<7 \%, \quad 7 \% \leq \mathrm{HbA} 1 \mathrm{c} \leq 9 \%, \quad$ and HbA1c $>9 \%$ as the judgment conditions. The final decision was made through different processes following the rule below.

The rule of the decision tree was developed based on the 2017 Chinese T2DM guideline [10] and 2018 Chinese T2DM guideline [11] for primary care. Expert consensus and clinical experience were used as supplements. In all patients with T2DM, lifestyle intervention is recommended as the fundamental treatment. If HbA1c is still over the target, which is $7 \%$, metformin monotherapy should be started on top of the lifestyle intervention unless there are contraindications. In patients with metformin contraindications or intolerance, consider an initial drug of alpha-glucosidase or insulin secretagogues. If the HbA1c target is not achieved after approximately 3 months, consider a combination of metformin and one of the four available treatment options: insulin secretagogues (sulfonylurea), thiazolidinedione, alphaglucosidase, or insulin (once/twice daily). If the HbA1c target is still not achieved after 3 months of dual therapy, proceed to triple therapy combined with three drugs with different mechanisms. Again, if the HbA1c target is not achieved after 3 months of triple therapy, refer the patient to higher level hospitals.

In addition, if the HbA1c of newly diagnosed T2DM patient is $>9 \%$ and the patient is markedly symptomatic, consider basal insulin injectable plus an oral antidiabetic drug (OAD) combination therapy or hospitalization for intensive glycemia control or refer the patient to a higher level hospital. If $\mathrm{HbA1c}$ is $>9 \%$, ketone body testing is recommended as well. When ketone bodies are present, patients should be referred to higher level hospitals.

\section{Clinical Decision Tree Outputs}

The decision tree followed eight classes of the medication catalogue of the T2DM primary care system in China, including metformin, alphaglucosidase, sulfonylurea (SU), glinides, thiazolidinedione (TZD), dipeptidyl peptidase-4 inhibitors (DDP-4i), and basal and premix insulin. It generated treatment recommendations or advised further referral. The multiple regimens under the decision tree could be selected as per the physician's discretion. In addition, the decision tree provided medication reminders or alerts, such as drug contraindications, principle of medication selection, and diabetic ketoacidosis alerts, to the primary care physician to reduce medication errors. 


\section{Validation of the Clinical Decision Tree}

\section{Real-World Datasets}

The decision tree was validated with data from two registry studies: the Newly Diagnosed Type 2 Diabetes (NEW2D) study and the Observational Registry of Basal Insulin Treatment (ORBIT) study $[12,13]$. The NEW2D study was a prospective cohort study in China. It had 5193 newly diagnosed T2DM patients with follow-up visits at every 3 months for up to 1 year, observing the effectiveness of the treatment paradigm in real-world practice. The ORBIT study was a multi-center, prospective registry study, recruiting 19,894 T2DM patients initiated with basal insulin, followed up at 3 and 6 months, which evaluated the effectiveness and safety of different basal insulins in a real-world setting in China. The participants in the ORBIT study and NEW2D study represent different patient populations, and the baseline HbA1c levels of the patients in the ORBIT study were higher than those in the NEW2D study $(9.6 \pm 2.0 \%$ vs. $8.4 \pm 2.5 \%) \quad[12,13]$. Participants in the ORBIT study might also have had more complications and needed more combination medication to control their diabetes, which was different from the situation of patients in the NEW2D study.

Both the NEW2D study and ORBIT study were registered at http://www.clinicaltrials.gov (NCT01525693 and NCT01859598). The NEW2D study obtained ethical approval from the Ethic Committees of Peking University People's Hospital. The protocol of ORBIT was approved by the Institutional Review Board (IRB) of Peking University. Informed consents for both studies were obtained before all study procedures. The studies were carried out in accordance with the Declaration of Helsinki 1964 and its later amendments.

\section{Generation of Cohort 1 and Cohort 2}

Since the NEW2D and ORBIT datasets recorded patient data every 3 months, we randomly selected any "3-month visit data" from each patient to construct an independent dataset (Cohort 1) to perform statistical analysis. For each patient, the HbA1c at a randomly selected current visit was defined as the "baseline HbA1c," and the value after 3 months was defined as the "HbA1c value at 3 months." We generated a random number as the index of the visit; the random seed in the cohort 1 analysis was 123 .

The data included 19,894 patients from ORBIT and 5193 patients from NEW2D. There were 23,848 patients included in the final analysis excluding 741 patients from ORBIT and 498 from NEW2D with missing features, such as the baseline HbA1c, HbA1c value at 3 months, or physician's prescription. The efficacy and safety outcomes were analyzed separately based on different patient numbers after excluding patients with corresponding missing values. For HbA1c analysis, 14,981 out of 23,848 patients were excluded because of missing HbA1c values at 3 months. In cohort 1 , only 8867 patients had HbA1c values at 3 months, so the HbA1crelated outcomes were analyzed in 8867 patients. For hypoglycemia analysis, 2778 out of 23,848 patients were excluded because of missing hypoglycemia data at 3 months. There were 21,070 patients with hypoglycemia results at 3 months, and then the hypoglycemia-related analysis was performed on 21,070 patients (Fig. 1).

As we know, the glycemia control rate of T2DM is low in China, and more attention should be paid to patients with baseline HbA1c $\geq 7 \%$ to improve their treatment outcome. We excluded patients with baseline HbA1c $<7 \%$ to further validate the clinical value of the decision tree at 3 months. There were 5788 patients with baseline HbA1c $\geq 7 \%$ involved in the HbA1c-related analysis and 17,943 patients involved in the hypoglycemiarelated analysis (Fig. 1).

Because cohort 1 was randomly selected, to confirm the reliability of our results, we constructed another independent dataset (cohort 2) with different random seed "789" in the same population. The data disposition of cohort 2 was similar to that of cohort 1 (Fig. 1).

\section{Generation of the Holistic Cohort}

The patients in both the NEW2D and ORBIT studies had regular clinical visits, which 


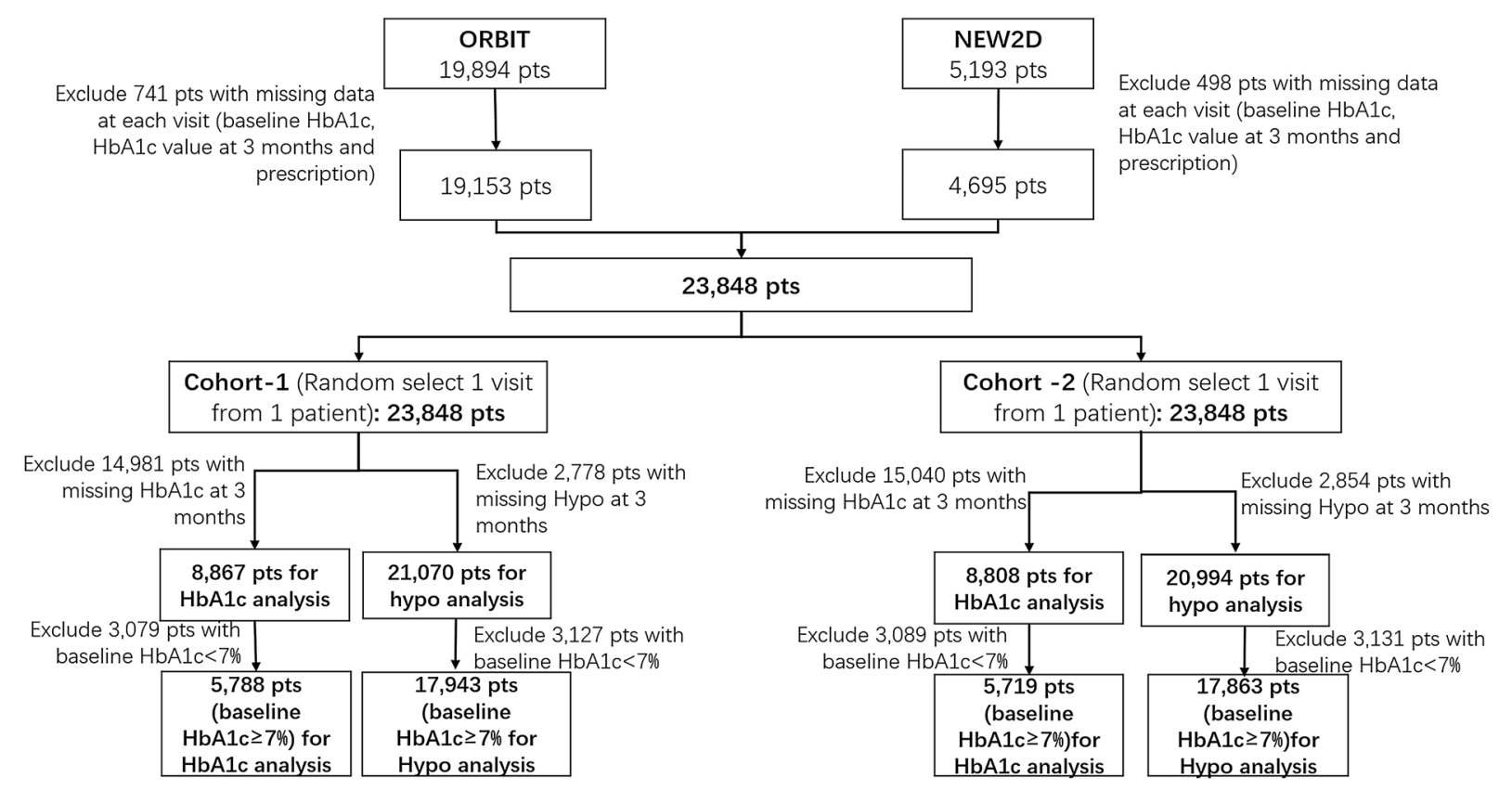

Fig. 1 Patient dispositions in the cohort 1 and cohort 2 datasets. ORBIT Observational Registry of Basal Insulin Treatment study, NEW2D Newly Diagnosed Type 2

Diabetes study, Pts patients, HbAlc glycosylated hemoglobin A1c, Hypo hypoglycemia

reflected real-world practice, and the previous treatment would have an impact on the following treatment's effectiveness. Therefore, the data from every 3-month visit from each patient were constructed into a "holistic cohort," which was composed of all data from NEW2D and ORBIT, and were real-world practice scenarios with repetitive visits.

There were 5193 patients from the NEW2D study, and each patient had five follow-up visits (recorded as v0, v1, v2, v3, v4) with 3-month duration between visits. Thus, we had 25,965 visits (5193 patients*5 visits) from the NEW2D study. Similarly, there were 19,894 patients from the ORBIT study with three visits (recorded as v0, v1, v2). So, we had 59,682 visits $(19,894$ patients 3 visits) from the ORBIT study. Since the data fed into the decision tree should have the baseline HbA1c and medication information, we excluded 11,197 and 35,359 visits with missing values from the NEW2D and ORBIT study, respectively. After removing these missing data, there were 39,091 visits in the final "holistic cohort." In the same way, the efficacy and safety outcomes were analyzed

separately based on different sample numbers after excluding samples with corresponding missing values (Fig. 2).

\section{Study Grouping}

Since in both the ORBIT and NEW2D studies the medications and laboratory results (if available) at each visit were recorded, we could allocate the patients in each cohort (cohort 1 , cohort 2 and the holistic cohort) into the compliance group or non-compliance group by judging whether the physician's prescription was consistent with the recommendations from the decision tree or not.

In the real-world setting, the medication might be changed based on the physician's prescription within the 3-month interval, so we set up the rules to define the baseline treatment regimen as below:

1. The add-on/changed medication was considered as a member of the baseline treatment regimen if its usage was $\geq 1$ month; it 




Fig. 2 Patient disposition of the holistic cohort dataset. ORBIT Observational Registry of the Basal Insulin Treatment study, NEW2D Newly Diagnosed Type 2

was supposed that medication had an impact on HbA1c after 1 month of usage.

2. The add-on insulin in the ORBIT data set was considered as a member of the baseline treatment regimen irrespective of the usage duration because the insulin initiation was mandatary per the ORBIT study protocol.

\section{Study Outcomes}

The primary outcome was the difference in HbA1c change at 3 months from baseline between the two groups. The secondary outcomes included the difference in the proportion of patients achieving $\mathrm{HbA} 1 \mathrm{c}<7 \%$ at 3 months between the two groups and the difference in the incidence of self-reported hypoglycemia at 3 months.

We also evaluated the above clinical outcomes in patients with baseline $\mathrm{HbA} 1 \mathrm{c} \geq 7 \%$. In addition, we further detected the difference in the proportion of patients (baseline HbA1c

Diabetes study, Pts patients, HbAlc glycosylated hemoglobin Alc, Hypo hypoglycemia

$\geq 7 \%$ ) with a HbA1c reduction $\geq 0.3 \%$, which was recommended as a non-inferiority margin and considered to be clinically meaningful by the FDA $[14,15]$.

\section{Statistical Analysis}

In cohort 1 , the difference in the mean HbA1c change between the two groups was analyzed using linear regression with inverse probability of treatment weighting (IPTW) with adjustment for age, gender, body mass index (BMI), patients' education level, diabetes duration, baseline HbA1c, diabetes complications, systolic blood pressure (SBP), and exercise.

The differences in the proportion of patients achieving $\mathrm{HbA} 1 \mathrm{c}<7 \%$ at 3 months and HbA1c reduction $\geq 0.3 \%$ at 3 months from baseline between the two groups were analyzed using logistic regression with IPTW with adjustment for age, gender, BMI, patients' education level, 
Table 1 Baseline characteristics

\begin{tabular}{|c|c|c|c|c|}
\hline Variable & $\begin{array}{l}\text { Total } \\
n=23,848\end{array}$ & $\begin{array}{l}\text { Non-compliance } \\
\text { group } \\
n=8955\end{array}$ & $\begin{array}{l}\text { Compliance group } \\
n=14,893\end{array}$ & $P$ value \\
\hline Age (years) & $56.0(10.8)$ & $55.9(11.0)$ & $56.0(10.8)$ & $<0.411$ \\
\hline Gender (male/female) & $\begin{array}{l}12,599 / 11249(52.8 \% / \\
47.2 \%)\end{array}$ & $\begin{array}{l}4699 / 4256(52.5 \% / \\
47.5 \%)\end{array}$ & $\begin{array}{l}7900 / 6993(53.0 \% / \\
47.0 \%)\end{array}$ & 0.399 \\
\hline $\operatorname{BMI}\left(\mathrm{kg} / \mathrm{m}^{2}\right)$ & $24.8(3.4)$ & $24.6(3.4)$ & $24.9(3.4)$ & $<0.001$ \\
\hline HbAlc (\%) & $9.0(2.2)$ & $8.9(2.1)$ & $9.1(2.2)$ & $<0.001$ \\
\hline $\mathrm{SBP}(\mathrm{mmHg})$ & $129.6(15.5)$ & $129.9(15.8)$ & $129.5(15.3)$ & 0.038 \\
\hline Education & & & & $<0.001$ \\
\hline Below primary school & $6083(25.5 \%)$ & $2452(27.4 \%)$ & $3631(24.4 \%)$ & \\
\hline Junior high school & $7270(30.5 \%)$ & $2798(31.2 \%)$ & $4472(30.0 \%)$ & \\
\hline Senior high school & $5967(25.0 \%)$ & $2130(23.8 \%)$ & $3837(25.8 \%)$ & \\
\hline Junior college & $2617(11.0 \%)$ & $921(10.3 \%)$ & $1695(11.4 \%)$ & \\
\hline Undergraduate or above & $1907(8.0 \%)$ & $654(7.3 \%)$ & $1253(8.4 \%)$ & \\
\hline $\begin{array}{l}\text { Duration of diabetes (years) (median } \\
{[\text { [QR }] \text { ) }}\end{array}$ & $3.94[0.70,8.58]$ & $3.39[0.54,8.39]$ & $4.14[0.77,8.69]$ & $<0.001$ \\
\hline Regular exercise & $16,298(68.9 \%)$ & $5903(66.5 \%)$ & $10,395(70.4 \%)$ & $<0.001$ \\
\hline Diabetic complication & $7546(31.6 \%)$ & $2959(33.0 \%)$ & $4587(30.8 \%)$ & $<0.001$ \\
\hline History of hypoglycemic before visit & $1476(6.2 \%)$ & $643(7.2 \%)$ & $833(5.6 \%)$ & $<0.001$ \\
\hline
\end{tabular}

Continuous variables were summarized as means $(\mathrm{SD})$ or interquartile range $[\mathrm{IQR}]$; categorical variables were summarized as the number of patients with corresponding percentage $n(\%) . P<0.05$ was considered statistically significant. HbA1c glycosylated hemoglobin A1c, SBP systolic blood pressure, BMI body mass index, Regular Exercise exercise $>5$ times a week, and the amount of exercise each time is equivalent to half an hour fast walking, Non-compliance group: decision tree noncompliance group, Compliance group: decision tree compliance group

diabetes duration, baseline $\mathrm{HbA1c}$, diabetes complications, SBP, and exercise.

The difference in the incidence of self-reported hypoglycemia at 3 months was analyzed using logistic regression with IPTW, with adjustment for age, gender, BMI, patients' education level, diabetes duration, baseline HbA1c, diabetes complications, SBP, exercise, and history of hypoglycemia.

The statistical analysis of cohort 2 was performed repetitively like in cohort 1 .

All analyses were performed using the $\mathrm{R}$ Project for Statistical Computing (version 3.5.1) (hereafter referred to as R). $P<0.05$ was considered statistically significant.

\section{Sensitivity Analysis}

The holistic cohort was used to perform the sensitivity analysis. In the holistic cohort, the generalized estimating equation (GEE) with the IPTW method was applied with adjustment for confounders including age, gender, BMI, patients' education level, diabetes duration, baseline HbA1c, diabetes complications, SBP, and exercise. All analyses were performed using 
Table 2 Statistical analysis of the cohort 1 dataset

\begin{tabular}{clclllc}
\hline Outcome & $\begin{array}{l}\text { Decision tree- } \\
\text { compliance }\end{array}$ & $\begin{array}{l}\text { Number of } \\
\text { samples }\end{array}$ & $\begin{array}{l}\text { Incidence of } \\
\text { outcome }\end{array}$ & Mean (95\% CI) & $\begin{array}{l}\text { Adjusted OR } \\
(\mathbf{9 5 \%} \text { CI) }\end{array}$ & $\begin{array}{l}\text { Adjusted } \\
\boldsymbol{P} \text { value }\end{array}$ \\
\hline $\begin{array}{c}\text { HbAlc } \\
\text { change }\end{array}$ & Yes & 4490 & - & -0.21 & - & $<0.001$ \\
No & 4377 & - & $(-0.29$ to -0.14$)$ & $1.7(1.6,1.9)$ & $<0.001$ \\
HbAlc $<7 \%$ & Yes & 4490 & $2744(61.1 \%)$ & & & \\
& No & 4377 & $1939(44.3 \%)$ & - & $0.7(0.66,0.8)$ & $<0.001$ \\
Hypoglycemia & Yes & 13,252 & $935(7.1 \%)$ & - & & \\
& No & 7818 & $731(9.4 \%)$ & & & \\
\hline
\end{tabular}

Continuous variables were summarized as means ( $95 \% \mathrm{CI})$; categorical variables were summarized as the number of patients with corresponding percentage $n$ (\%). $P<0.05$ was considered statistically significant. Non-compliance group: decision tree non-compliance group; Compliance group: decision tree compliance group. HbAlc glycosylated hemoglobin A1c

R (version 3.5.1). $P<0.05$ was considered statistically significant.

\section{RESULTS}

\section{Baseline Characteristics}

A total of 23,848 patients with T2DM were finally enrolled in the study $(52.8 \%$ males and $47.2 \%$ females, mean age: $56.0 \pm 10.8$ years). The baseline HbA1c was $9.1 \pm 2.2 \%$ in the compliance group vs. $8.9 \pm 2.1 \%$ in the noncompliance group, and the ages were $56.0 \pm 10.8$ years vs. $55.9 \pm 11.0$ years, respectively. Compared with those in the compliance group, patients in the non-compliance group had significantly lower BMIs, lower HbA1c values at baseline, shorter diabetes duration, lower education levels, and lower exercise levels, but higher SBP. They were also significantly more likely to have diabetic complications and a history of hypoglycemia. Table 1 summarizes the baseline characteristics of the patients in each group.

\section{Clinical Outcomes}

In cohort 1 , the $\mathrm{HbA1c}$ reduction at 3 months was $0.9 \%$ in the compliance group vs. $0.8 \%$ in the non-compliance group (mean difference:
- $0.21 \%$ [95\% CI -0.29 to -0.14$] ; P<0.001$ ). The compliance group had a statistically significantly higher proportion of patients achieving HbA1c $<7 \%$ at 3 months compared to the non-compliance group $(61.1 \%$ vs. $44.3 \%$, OR 1.7; [95\% CI 1.6-1.9]; $P<0.001$ ). Hypoglycemic events occurred in $7.1 \%$ of patients in the compliance group, which was significantly lower than that of $9.4 \%$ in the non-compliance group (OR 0.7; [95\% CI 0.66-0.8]; $P<0.001$ ) (Table 2).

In patients with baseline $\mathrm{HbA} 1 \mathrm{c} \geq 7 \%$, the baseline HbA1c was $9.6 \pm 2.1 \%$ in the compliance group vs. $8.6 \pm 1.7 \%$ in the non-compliance group. The $\mathrm{HbA1c}$ reduction from baseline to 3 months was $2.1 \%$ in the compliance group vs. $1.1 \%$ in the non-compliance group (mean difference $-0.37 \%$; [95\% CI -0.44 to -0.30 ]; $P<0.001)$. The compliance group was also more likely to achieve HbA1c $<7 \%$ at 3 months compared to the non-compliance group after adjusting for confounding factors (OR 1.3; [95\% CI 1.2-1.5]; $P<0.001)$. Furthermore, the compliance group had a greater percentage of patients with $\mathrm{HbA1c}$ reduction $\geq 0.3 \%$ at 3 months than the non-compliance group among patients who had baseline HbA1c $\geq 7 \%$ (85.1\% vs. $66.5 \%$, OR 1.9; [95\% CI $1.7-2.2]$; $P<0.001)$. Hypoglycemic events occurred in $7.9 \%$ of patients in the compliance group, which was significantly lower than that of $9.6 \%$ 
Table 3 Statistical analysis in patients with baseline $\mathrm{HbAlc} \geq 7 \%$ in the cohort 1 dataset

\begin{tabular}{llclclc}
\hline Outcome & $\begin{array}{l}\text { Decision tree- } \\
\text { compliance }\end{array}$ & $\begin{array}{l}\text { Number of } \\
\text { samples }\end{array}$ & $\begin{array}{l}\text { Incidence of } \\
\text { outcome }\end{array}$ & Mean (95\% CI) & $\begin{array}{l}\text { Adjusted OR } \\
(\mathbf{9 5 \%} \text { CI })\end{array}$ & $\begin{array}{l}\text { Adjusted } \\
\boldsymbol{P} \text { value }\end{array}$ \\
\hline HbAlc change & Yes & 2158 & - & $-0.37(-0.44$ & - & $<0.001$ \\
& No & 3630 & - & to -0.30$)$ & & \\
HbAlc $<7 \%$ & Yes & 2158 & $778(36.1 \%)$ & - & $1.3(1.2,1.5)$ & $<0.001$ \\
& No & 3630 & $1350(37.2 \%)$ & & $1.9(1.7,2.2)$ & $<0.001$ \\
HbAlc & Yes & 2158 & $1837(85.1 \%)$ & - & & \\
reduction $\geq 0.3 \%$ & No & 3630 & $2415(66.5 \%)$ & $0.7(0.66,0.8)$ & $<0.001$ \\
Hypoglycemia & Yes & 10,885 & $859(7.9 \%)$ & - & & \\
\hline
\end{tabular}

Continuous variables were summarized as means $(95 \% \mathrm{CI})$; categorical variables were summarized as the number of patients with corresponding percentage $n(\%) . P<0.05$ was considered statistically significant. Non-compliance group: decision tree non-compliance group; Compliance group: decision tree compliance group. HbAlc glycosylated hemoglobin A1c

in the non-compliance group (OR 0.7; [95\% CI 0.66-0.8]; $P<0.001$ ) (Table 3).

In cohort 2 , similar results to those of the cohort 1 analysis were observed, as shown in Appendix supplemental Table 1 and 2. The $\mathrm{HbA1c}$ reduction from baseline to 3 months was $0.9 \%$ in the compliance group vs. $0.84 \%$ in the non-compliance group (mean difference: $-0.17 \% ; \quad[95 \%$ CI -0.25 to -0.10$]$; $P<0.001)$. In the compliance group $61.1 \%$ of patients achieved the goal of $\mathrm{HbA} 1 \mathrm{c}<7 \%$ in 3 months compared to $44.4 \%$ in the non-compliance group (OR 1.7; [95\% CI 1.5-1.8]; $P<0.0001)$. Hypoglycemic events occurred in $7.1 \%$ of patients in the compliance group, which was significantly lower than the $9.1 \%$ found in the non-compliance group (OR 0.8; [95\% CI 0.7-0.8]; $P<0.001$ ).

In patients with baseline $\mathrm{HbA} 1 \mathrm{c} \geq 7 \%$, the HbA1c reduction at 3 months was $2.0 \%$ in the compliance group vs. $1.1 \%$ in the non-compliance group (mean difference: - 0. 31\%; [95\% CI -0.38 to -0.24$] ; P<0.001)$. The compliance group was also more likely to achieve HbA1c $<7 \%$ at 3 months compared to the noncompliance group after adjusting for confounding factors (OR 1.3; [95\% CI 1.1-1.4]; $P<0.001)$. Furthermore, the compliance group had a greater percentage of patients with $\mathrm{HbA1c}$ reduction $\geq 0.3 \%$ at 3 months from baseline than the non-compliance group $(85.0 \%$ vs. 66.4\%, OR, 2.0 [95\% CI 1.7-2.2]; $P<0.001$ ). The hypoglycemic events occurred in $7.9 \%$ of patients in the compliance group, which was significantly lower than that of $9.4 \%$ in the non-compliance group (OR 0.8; [95\% CI 0.7-0.84]; $P<0.001)$. In patients with baseline HbA1c $<7 \%$ in both cohort 1 and 2, there were similar trends of the compliance group having a greater $\mathrm{HbA1c}$ reduction from baseline, a higher percentage of patients maintaining $\mathrm{HbA} 1 \mathrm{c}<$ $7 \%$, and less hypoglycemia, but overall, they were not significantly different (data shown in Appendix supplemental Table 3 and 4).

\section{Sensitivity Analysis}

In the holistic cohort, $66.8 \%$ of patients in the compliance group achieved $\mathrm{HbA} 1 \mathrm{c}<7 \%$ at 3 months but only $44.0 \%$ in the non-compliance group (OR 1.1; [95\% CI $1.07-1.2]$; $P<0.001)$. In the compliance group, $6.5 \%$ experienced hypoglycemic events, which was significantly lower than the $8.2 \%$ in the noncompliance group (OR 0.84; [95\% CI 0.78-0.9]; 
Table 4 Statistical analysis of the holistic cohort dataset

\begin{tabular}{clllccc}
\hline Outcome & $\begin{array}{l}\text { Decision tree- } \\
\text { compliance }\end{array}$ & $\begin{array}{l}\text { Number of } \\
\text { samples }\end{array}$ & $\begin{array}{l}\text { Incidence of } \\
\text { outcome }\end{array}$ & Mean (95\% CI) & $\begin{array}{l}\text { Adjusted OR } \\
(95 \% \text { CI })\end{array}$ & $\begin{array}{l}\text { Adjusted } \\
\boldsymbol{P} \text { value }\end{array}$ \\
\hline $\begin{array}{c}\text { HbAlc } \\
\text { change }\end{array}$ & Yes & 12,097 & - & -0.03 & - & 0.067 \\
No & 11,083 & - & $(-0.06-0.00)$ & & \\
HbAlc $<7 \%$ & Yes & 12,097 & $8078(66.8 \%)$ & - & $1.1(1.07,1.2)$ & $<0.001$ \\
& No & 11,083 & $4872(44.0 \%)$ & & \\
Hypoglycemia & Yes & 20,909 & $1361(6.5 \%)$ & - & $0.84(0.78,0.9)$ & $<0.001$ \\
& No & 14,561 & $1195(8.2 \%)$ & & \\
\hline
\end{tabular}

Continuous variables were summarized as means $(95 \% \mathrm{CI})$; categorical variables were summarized as the number of patients with corresponding percentage $n(\%) . P<0.05$ was considered statistically significant. Non-compliance group: decision tree non-compliance group; Compliance group: decision tree compliance group. HbAlc glycosylated hemoglobin Alc

$P<0.001)$. Although the HbA1c reduction at 3 months in the compliance group was less than that in the non-compliance group after confounder adjustment, the difference between the two groups was not significant $(0.6 \%$ vs. $0.9 \%$; mean difference $-0.03 \%$; [95\% CI -0.06 to 0.00]; $P=0.067$ ) (Table 4).

\section{DISCUSSION}

In our study, we developed a guideline-based decision tree to help primary care physicians give standard care to patients with T2DM in China according to the Chinese guideline. In our decision tree validation analysis, we found that the patients in the decision tree compliance group had a greater $\mathrm{HbA1c}$ reduction at 3 months, were more likely to achieve the goal of $\mathrm{HbA} 1 \mathrm{c}<7 \%$, and were less likely to experience a hypoglycemic event in 3 months. In patients with baseline $\mathrm{HbA} 1 \mathrm{c} \geq 7 \%$, similar results were observed. Furthermore, more patients with baseline HbA1c $\geq 7 \%$ achieved a HbA1c reduction $>0.3 \%$ in the compliance group. This indicated that the output of the clinical decision tree would help T2DM patients have better HbA1c control with less risk of hypoglycemia. The effectiveness of the decision tree was well tested with the real-world data. An earlier study [16] also showed that a computerized decision supporting reminder, which was similar to our decision tree in Primary Care for
Type 2 Diabetes, improved patients' outcomes, associated with a significant $\mathrm{HbA1c}$ reduction of $0.3 \%$ from baseline.

In our study, the decision tree was designed based on the Chinese T2DM guideline and provided guideline-concordant treatment recommendations to physicians. The reason why the decision tree compliance group had much better glycemic control than the non-compliance group was that following the recommendation of the decision tree meant better compliance with the Chinese guideline following the standard treatment procedure. The Chinese guideline was well recognized as the most authoritative evidence-based source for diabetes care in China. We believe that the Chinese T2DM guideline will provide standard of care management to physicians and eventually benefit more patients. This assumption was also verified by our previous work [17]. Patients with guideline-concordant treatment regimens had a significantly higher proportion $(80.5 \%)$ of patients achieving FPG $<7.0 \mathrm{mmol} / \mathrm{l}$ compared to $53.5 \%$ in those with guideline non-concordant treatment regimens $(P<0.001)$. These findings also indicated that guideline adherence will play an important role in improving diabetes management.

It is believed that computerized decision support tools can help improve guideline adherence. Computerized knowledge management-related studies at the physician level [18] 
showed that physicians had 71-227\% higher compliance with recommended diabetes care procedures in the prompted group than in the control groups. Another study $[19,20]$ showed that computerized decision support based on a clinical practice guideline improved compliance with care standards, with $32.0 \%$ and $15.6 \%$ in the intervention and control group, respectively $(P=0.01)$. In addition, a study [21] using computer-generated individualized e-mails to physicians feeding back their adherence to diabetes guidelines also showed the overall compliance improved to $35.3 \%$ in the intervention group vs. $6.1 \%$ in the control group $(P<0.01)$. A computerized decision support tool is important because traditional clinical guidelines have been shown to be difficult to implement in practice with low clinician adherence $[22,23]$. The assumption that practitioners will read, internalize, and implement guidelines does not hold true [24]. However, the rules implicitly defined in guidelines can be literally encoded into the clinical decision tree. Wagner et al. $[25,26]$ also noted that ideally effective models for chronic disease care emphasize decision support tools that can be used to guide evidence-based, guideline-concordant care which is consistent with patient needs and preferences. In conclusion, the decision tree is such a decision support tool providing fundamentals of the Diabetes Clinical Decision Support System (CDSS) for primary care, improving guideline compliance and achieving better diabetes management.

However, we should also note that diabetes management should not only be glucose centered; multifactorial management is important. In our current version of the decision tree, we only give suggestions for an algorithm for antihyperglycemic treatment. We hope we can add more information about lifestyle interventions, hypertensive control, and lipid treatment to our decision tree in the future.

\section{Strengths and Limitations}

This study had some strengths. On one hand, this study used two randomly selected datasets to validate the analysis results. On the other, we used not only randomly selected data, but also sampling at the patient visit level, which mimics the real-world setting in which patients go to clinics continuously at regular intervals. All the analyses showed consistent results and made our conclusions more robust. The patients in the compliance group were more likely to achieve HbA1c $<7 \%$, more likely to have a HbA1c reduction $\geq 0.3 \%$ from baseline, and less likely to experience hypoglycemia at 3 months. This indicated that the decision tree can be used in broad T2DM populations and continuously to help patients achieve better glycemic control with fewer hypoglycemic risks.

However, this study also had some limitations. First, it was retrospective and observational with a heterogeneous patient population, and the baseline characteristics were not matched. Second, the medications might be changed per physician's prescription within the 3 -month interval. This may have some crossover effects on glycemic control or hypoglycemia incidence. Moreover, a method to monitor the success of advice given in the guideline-based decision tree was also needed.

\section{CONCLUSION}

The patients in the compliance group had a greater HbA1c reduction at 3 months from baseline and were more likely to achieve HbA1c $<7 \%$, more likely to have HbA1c reduction $\geq 0.3 \%$ from baseline, and less likely to experience hypoglycemia at 3 months. The decision tree can aid physicians, especially those in primary care, in helping their patients reach their glycemic targets with fewer hypoglycemic risks.

\section{ACKNOWLEDGEMENTS}

Funding. The study and the Journal's Rapid Service Fee were funded by Sanofi (China) Investment, Shanghai, China.

Authors' Contributions. Y.Y.L., H.W.: designed the research, performed data 
interpretation, edited and revised the article. L.N.J., N.C., A.H.L.: helped design the study, performed data interpretation, reviewed and revised the article, authored the article. X.Y.L., T.T.Z.: performed data acquisition, analyzed data, edited and revised the article. X.Z.S., X.Z., H.Q.Y.: helped with data interpretation, reviewed and revised the article. P.H.Z., Y.H.H.: performed data acquisition, reviewed and revised the article. L.N.J.: edited and reviewed the final article and was the principal investigator of both the NEW2D and ORBIT studies. All named authors met the International Committee of Medical Journal Editors (ICMJE) criteria for authorship for this article, took responsibility for the integrity of the work as a whole, and gave their approval for this version to be published. The authors acknowledge all subjects who participated in the clinical trials. The authors also acknowledge the staff at the VitalStrategic Research Institute for their contributions to this study.

Disclosures. Yingying Luo, Hong $\mathrm{Wu}$, Xiyang Liao, Tingting Zhao, Nan Cui, Aihua Li, Xingzhi Sun, Puhong Zhang, Yahua Huang, Xia Zhang, Huiqiu, Yin and Linong Ji have nothing to disclose.

Compliance with Ethics Guidelines. Both the NEW2D study and ORBIT study were registered at http://www.clinicaltrials.gov (NCT01525693 \& NCT01859598). The NEW2D study obtained ethical approval from the Ethic Committees of Peking University People's Hospital. The ORBIT protocol was approved by the Institutional Review Board (IRB) of Peking University. Informed consents from patients in both studies were obtained before all study procedures. The studies were carried out in accordance with the Declaration of Helsinki 1964 and its later amendments.

Data Availability. The datasets analyzed during the current study are not publicly available because of Institutional Review Board limitations but are available from the corresponding author on reasonable request.
Open Access. This article is licensed under a Creative Commons Attribution-NonCommercial 4.0 International License, which permits any non-commercial use, sharing, adaptation, distribution and reproduction in any medium or format, as long as you give appropriate credit to the original author(s) and the source, provide a link to the Creative Commons licence, and indicate if changes were made. The images or other third party material in this article are included in the article's Creative Commons licence, unless indicated otherwise in a credit line to the material. If material is not included in the article's Creative Commons licence and your intended use is not permitted by statutory regulation or exceeds the permitted use, you will need to obtain permission directly from the copyright holder. To view a copy of this licence, visit http://creativecommons.org/licenses/by$\mathrm{nc} / 4.0 /$.

\section{REFERENCES}

1. Hills AP, et al. Epidemiology and determinants of type 2 diabetes in South Asia. Lancet Diabetes Endocrinol. 2018;6:966-78.

2. Wang L, et al. Prevalence and ethnic pattern of diabetes and prediabetes in China in 2013. JAMA. 2017;317:2515-23.

3. Chatterjee $\mathrm{S}$, et al. Type 2 diabetes. Lancet (London, England). 2017;389:2239-51.

4. Weiping T, et al. Prevalence of diabetes recorded in mainland China using 2018 diagnostic criteria from the American Diabetes Association: national crosssectional study. BMJ. 2020;369:m997.

5. Liu M, et al. Burden of diabetes, hyperglycaemia in China from to 2016: findings from the 1990 to 2016, global burden of disease study. Diabet Metab. 2019;45(3):286-93.

6. https://www.who.int/healthpromotion/conferen ces/9gchp/healthy-china/en/. Accessed Apr 28, 2021.

7. http://www.gov.cn/zhengce/content/2015-09/11/ content_10158.htm. Accessed Apr 28, 2021.

8. Meng $\mathrm{Yu}$, et al. Examining the multi-scalar unevenness of high-quality healthcare resources 
distribution in China. Int J Environ Res Public Health. 2019;16:2813.

9. Li Xi, et al. Quality of primary health care in China: challenges and recommendations. Lancet. 2020;395:1802-12.

10. Chinese Diabetes Society. China Type 2 Diabetes Treatment Guideline (2017). Chin J Diabetes Mellitus. 2018;10(1):4-67.

11. Chinese Diabetes Society. National guidelines for the prevention and control of diabetes in primary care (2018). Chin J Intern Med. 2018;57(12): 885-93.

12. Cai X, et al. Evaluation of effectiveness of treatment paradigm for newly diagnosed type 2 diabetes patients in Chin: a nationwide prospective cohort study. J Diabetes Investing. 2020;11(1):151-61.

13. Ji L, et al. Observational Registry of Basal Insulin Treatment (ORBIT) in patients with type 2 diabetes uncontrolled by oral hypoglycemic agents in China-study design and baseline characteristics. Diabetes Technol Ther. 2015;17(10):735-44.

14. US Food and Drug Administration. Center for Drug Evaluation and Research. Guidance for industry. Diabetes Mellitus: developing drugs and therapeutic biologics for treatment and prevention. 2008. http://docplayer.net/5967402-guidance-for-industy -diabetes-mellitus-developing-drugs-and-therapeu tic-biologics-for-treatment-and-prevention.html. Accessed Apr 28, 2021.

15. Garber AJ. Treat-to-target trials: uses, interpretation and review of concepts. Diabetes Obes Metab. 2014;16(3):193-205.

16. Phillips LS, Ziemer DC, Doyle JP, Barnes CS, Kolm P, Branch WT, Caudle JM, Cook CB, Dunbar VG, ElKebbi IM, Gallina DL, Hayes RP, Miller CD, Rhee MK, Thompson DM, Watkins C. An endocrinologist-supported intervention aimed at providers improves diabetes management in a primary care site: Improving Primary Care of African Americans with Diabetes (IPCAAD) 7. Diabetes Care. 2005;28: 235-60.
17. Sun $X$, et al. Integrating clinical knowledge and real-world evidence for type 2 diabetes treatment. AMIA Annu Symp Proc. 2020;4(2019):838-47.

18. Andrew Balas E, et al. Computerized knowledge management in diabetes care. Med Care. 2004;42: 610-21.

19. Lobach DF, et al. Computerized decision support based on a clinical practice guideline improves compliance with care standards. Am J Med. 1997;102:89-98.

20. Lobach DF, Hammond WE. Development and evaluation of a computer-assisted management protocol (CAMP): improved compliance with care guidelines for diabetes mellitus. In: Proceedings of the Annual Symposium on Computer Applications in Medical Care; 1994: pp. 787-91.

21. Lobach DF: Electronically distributed, computergenerated, individualized feedback enhances the use of a computerized practice guideline. In Cimino JJ (ed) AMIA Annual Fall Symposium. Philadelphia, PA: Hanley and Belfus, Inc.; 1996: pp. 493-7.

22. Davis DA, Taylor-Vaisey A. Translating guidelines into practice: a systematic review of theoretic concepts, practical experience and research evidence in the adoption of clinical practice guidelines. Can Med Assoc J. 1997;157:408-16.

23. Michael C, Rand CS, Powe NR, Wu AW, Wilson $\mathrm{MH}$. Why don' t physicians follow clinical practice guidelines? a framework for improvement. JAMA. 1999;282:1458-65.

24. Shortliffe, T. Medical thinking: what should we do? (2006). http://www.openclinical.org/medicalThink ing2006Summary2.html. Accessed 5 Jan 2021.

25. Schmittdie JA, et al. Population health management for diabetes: health care system-level approaches for improving quality and addressing disparities. Curr Diab Rep. 2017;17(5):31.

26. Wagner Chronic Care Model. http://www. improvingchroniccare.org/index.php? $\mathrm{p}=1$ : Models\&s=363. Accessed Dec 19, 2016. 